\title{
Analysis of MIMO Precoding with Base Station Cooperation and Imperfect Channel Estimation
}

\author{
Velser D. B. Correia, Francisco R. P. Cavalcanti, and Yuri C. B. Silva
}

\begin{abstract}
In this paper, the performance of different linear and non-linear precoding techniques is assessed assuming base station cooperation and imperfect channel estimation. The evaluation considers the downlink of a multicell-multiuser MIMO system in which the base stations cooperate as a joint MIMO transmitter, so that streams intended to all co-channel users are spread across all transmit antennas. Precoding techniques are employed to suppress co-channel interference and the average spectral efficiency per user is evaluated for different levels of perturbation, which is added to the actual channel. The present work shows that, depending on the antenna configuration and on the SNR, the algorithms may present a different sensitivity to estimation errors.
\end{abstract}

Keywords-Base Station Cooperation, MIMO, Precoding, Transmit Beamforming

\section{INTRODUCTION}

Multiple-input multiple-output (MIMO) systems are well known to boost capacity [1], [2] by providing multiple spatial dimensions and multiplexing gain. In these systems, capacity increases linearly with the minimum of transmit and receive antennas at high signal-to-noise-ratio (SNR).

Nevertheless, the achievement of such remarkable capacity gains for realistic multicell multiuser networks is still a hard challenge. MIMO structures in realistic scenarios with multiple users and aggressive frequency reuse leading to severe cochannel interference (CCI) may have their advantages belittled. Insufficient scattering and antenna spacing are serious practical issues regarding capacity gain achievements that can lead to rank-deficient and ill-conditioned MIMO channel matrices. The keyhole effect can be another cause of spatial correlation in some environments, therefore decreasing capacity even though each channel ends up with uncorrelated fading [3].

Some effort has been done in order to improve capacity in an interference-limited multicell multiuser scenario employing MIMO structures, like multiuser detection and turbo decoding [4], [5]. However, such receiver techniques improve the downlink system performance at the cost of increased receiver complexity which is seen to be the bottleneck for next-generation wireless systems. The natural idea to face this problem could be the translation of the CCI mitigation to the transmitter side, i.e., to the base station (BS), as in [6], [7], where more advanced signal processing is feasible.

The purpose of this paper is to evaluate joint precoding in a multicell multiuser MIMO system where perfect base station cooperation is assumed. Differently from the analysis done in [6], where perfect channel state information (CSI) at the

Velser D. B. Correia, Francisco R. P. Cavalcanti, and Yuri C. B. Silva, Departamento de Engenharia de Teleinformática, Universidade Federal do Ceará, Fortaleza, Brasil, E-mails: \{velser, rodrigo, yuri\}@gtel.ufc.br transmitter was considered, this work is intended to evaluate the performance of precoding techniques for different levels of channel estimation error, thus better approaching realistic scenarios. For reaching such an aim, both the performance (measured as the average spectral efficiency per user) and the error sensitivity of each precoder have been analyzed.

This paper is organized as follows. The next section describes the system model. Section III consists of a short review of the linear and nonlinear precoding algorithms implemented in this work, highlighting the main aspects of each one. Section IV describes the simulation procedure and analyzes the obtained results. Finally, in Section V, the conclusions are drawn.

\section{System Model}

The system considered in this work consists of 3 cells with 1 mobile station (MS) in each one. The 3 BSs cooperate in order to send channel information regarding their assigned users to a common central processing unit for a joint precoding design. No cooperation is available among co-channel MSs. Flat channel fading is assumed, such that inter-symbol interference can be disregarded, which is the case, for example, of multicarrier systems.

Let $N_{\mathrm{u}}$ denote the number of MSs, $N_{\mathrm{b}}$ the number of BSs, $N_{\mathrm{R}, i}$ the number of receive antennas of MS $i, N_{\mathrm{T}, j}$ the number of transmit antennas of BS $j, N_{\mathrm{R}}$ the total number of receive antennas and $N_{\mathrm{T}}$ the total number of transmit antennas. The assumption of perfect BS cooperation allows for conceiving the joint channel matrix $\mathbf{H} \in \mathbb{C}^{N_{\mathrm{R}} \times N_{\mathrm{T}}}$, which can be written as

$$
\mathbf{H}=\left[\begin{array}{cccc}
\mathbf{H}_{1,1} & \mathbf{H}_{1,2} & \cdots & \mathbf{H}_{1, N_{\mathrm{b}}} \\
\mathbf{H}_{2,1} & \mathbf{H}_{2,2} & \cdots & \mathbf{H}_{2, N_{\mathrm{b}}} \\
\vdots & \vdots & \ddots & \vdots \\
\mathbf{H}_{N_{\mathrm{u}}, 1} & \mathbf{H}_{N_{\mathrm{u}}, 2} & \cdots & \mathbf{H}_{N_{\mathrm{u}}, N_{\mathrm{b}}}
\end{array}\right]
$$

where $\mathbf{H}_{i, j} \in \mathbb{C}^{N_{\mathrm{R}, i} \times N_{\mathrm{T}, j}}$ contains the MIMO channel coefficients between $\mathrm{BS} j$ and MS $i$.

Assuming that the total number of data streams is equal to $N_{\mathrm{R}}$ and that $N_{\mathrm{T}} \geqslant N_{\mathrm{R}}$, the vector $\mathbf{y} \in \mathbb{C}^{N_{\mathrm{R}}}$ containing the received signal of all users is given by

$$
\mathbf{y}=\mathbf{G H T s}+\mathbf{G n},
$$

where $\mathbf{T} \in \mathbb{C}^{N_{\mathrm{T}} \times N_{\mathrm{R}}}$ is the joint precoding matrix, $\mathbf{G} \in$ $\mathbb{C}^{N_{\mathrm{R}} \times N_{\mathrm{R}}}$ represents the processing done at the receiver side, which can be a simple gain to recover the signals' original amplitudes or a more complex receive filter to cancel residual interference among streams of a same user, $\mathbf{s} \in \mathbb{C}^{N_{\mathrm{R}}}$ is the transmit symbol vector and $\mathbf{n} \in \mathbb{C}^{N_{\mathrm{R}}}$ is the additive white 
Gaussian noise. The system transmission/reception chain is illustrated in Fig. 1.

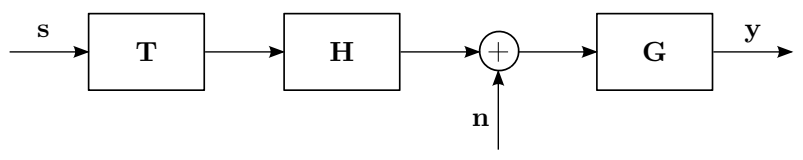

Fig. 1. MIMO system as a filter chain.

At the transmitter side only an estimate $\hat{\mathbf{H}} \in \mathbb{C}^{N_{\mathrm{R}} \times N_{\mathrm{T}}}$ of the actual channel matrix $\mathbf{H}$ is available. The matrix $\hat{\mathbf{H}}$ is modeled here as being the sum of the exact joint channel matrix $\mathbf{H}$ (whose entries take into account path-loss, shadowing and zero mean unit variance fast-fading combined) and a perturbation matrix $\mathbf{E}_{\mathrm{T}}$ with Gaussian distributed entries, which have the same mean value as those in $\mathbf{H}$ (due to path-loss and shadowing) and a variance $\sigma_{e}^{2}$, i.e.,

$$
\hat{\mathbf{H}}=\mathbf{H}+\mathbf{E}_{\mathrm{T}} \text {. }
$$

At the receiver side, depending on the considered receive processing algorithm, an estimate $\hat{\mathbf{H}}_{\mathrm{eq}} \in \mathbb{C}^{N_{\mathrm{R}} \times N_{\mathrm{R}}}$ of the equivalent channel $\mathbf{H}_{\mathrm{eq}}=\mathbf{H T} \in \mathbb{C}^{N_{\mathrm{R}} \times N_{\mathrm{R}}}$ may also be required. Since the focus of this paper is on the transmit processing techniques, perfect channel knowledge at the receivers is assumed, i.e., $\hat{\mathbf{H}}_{\mathrm{eq}}=\mathbf{H}_{\mathrm{eq}}$.

The joint precoding matrix $\mathbf{T}$, which is designed based on the estimated channel matrix $\hat{\mathbf{H}}$, can be seen as a product of the following two matrices:

$$
\mathbf{T}=\mathbf{P} \boldsymbol{\Omega},
$$

where $\mathbf{P} \in \mathbb{C}^{N_{\mathrm{T}} \times N_{\mathrm{R}}}$ is the matrix which actually performs the precoding technique and $\Omega \in \mathbb{R}^{N_{\mathrm{R}} \times N_{\mathrm{R}}}$ is a matrix that may perform a more accurate power allocation or just a power normalization. The design of the $\mathbf{P}$ matrix assumes a pooled power constraint which is not realistic, since each base station has its own power constraint. The model considered here $\boldsymbol{\Omega}$ assumes a simple power normalization taking into account perbase power constraints. This leads to a suboptimal approach, but which is more realistic than just assuming $\mathbf{T}=\mathbf{P}$ with pooled power constraint. For the present work, as in [6], $\Omega$ is defined as

$$
\boldsymbol{\Omega}=\left(\min _{j=1,2, \ldots, N_{\mathrm{b}}} \sqrt{\frac{P_{j}}{\left\|\mathbf{P}^{[j]}\right\|_{\mathrm{F}}^{2}}}\right) \mathbf{I},
$$

where $P_{j}$ is the power constraint of BS $j, \mathbf{P}^{[j]} \in \mathbb{C}^{N_{\mathrm{T}, j} \times N_{\mathrm{R}}}$ is the matrix obtained considering the rows of $\mathbf{P}$ corresponding to $\mathrm{BS} j,\|\cdot\|_{\mathrm{F}}$ is the Frobenius norm of a matrix, and $\mathbf{I}$ is an identity matrix of proper dimensions.

\section{LINEAR AND NON-LiNEAR PRECODING}

The design of $\mathbf{P}$ in (4) relies on the suitable choice of a precoding technique. In this section, some precoding techniques are very briefly reviewed.

Zero-Forcing (ZF) and Minimum Mean Square Error (MMSE) are both channel inversion-based precoders that can have their concepts concatenated in a single equation, such as

$$
\mathbf{P}=\mathbf{H}^{H}\left(\mathbf{H H}^{H}+\alpha \mathbf{I}\right)^{-1} .
$$

Zero-forcing corresponds to the case where $\alpha=0$, whereas for MMSE $\alpha$ assumes a value that is inversely proportional to the SNR. The idea of the regulation factor $\alpha$ is to properly affect the eigenvalues of $\mathbf{H H}^{H}$ in order to improve matrix conditioning. MMSE offers higher signal levels compared to the ZF approach $(\alpha=0)$. For both these algorithms a scalar receive filter corresponding to a simple gain is assumed. More details on these techniques can be found in [8].

The Channel Block-Diagonalization through Null Space Decomposition (BDNSD) consists of block-diagonalizing the joint channel so as to cancel all multiuser interference. Since the diagonalization is done block-wise, interference among individual streams of each user still remains and must be treated at the receiver side. The receive filter $\mathbf{G}$ can be implemented, in this case, as a zero-forcing filter.

For this method the precoder $\mathbf{P}$ is given by

$$
\mathbf{P}=\left[\begin{array}{lllll}
\mathbf{P}_{1} & \mathbf{P}_{2} & \mathbf{P}_{3} & \ldots & \mathbf{P}_{N_{\mathrm{u}}}
\end{array}\right],
$$

where $\mathbf{P}_{i} \in \mathbb{C}^{N_{\mathrm{T}} \times N_{\mathrm{R}, i}}$ is the transmit filter for MS $i$. Each precoder $\mathbf{P}_{i}$ can be written as

$$
\mathbf{P}_{i}=\tilde{\mathbf{V}}_{\text {null }, i} \mathbf{V}_{\text {signal }, i}
$$

where $\tilde{\mathbf{V}}_{\text {null, } i}$ is a matrix whose columns form a basis to the null-space of $\tilde{\mathbf{H}}_{\mathbf{i}} \in \mathbb{C}^{\left(N_{\mathrm{R}}-N_{\mathrm{R}, i}\right) \times N_{\mathrm{T}}}$, which is a joint channel matrix that comprises channels of all users other than $i$, i.e., $\{k \mid k \neq i\}$, and $\mathbf{V}_{\text {signal, } i}$ is a matrix whose columns form a basis to the signal space of the virtual channel $\mathbf{H}_{\mathrm{virtual}, i}$ seen by user $i$

$$
\mathbf{H}_{\mathrm{virtual}, i}=\mathbf{H}_{i} \tilde{\mathbf{V}}_{\mathrm{null}, i},
$$

where $\mathbf{H}_{i} \in \mathbb{C}^{N_{\mathrm{R}, i} \times N_{\mathrm{T}}}$ is a joint channel matrix regarding only user $i$ (a channel matrix between receive antennas of user $i$ and all transmit antennas of all BSs). For further details on block-diagonalization methods the interested reader can go to [9].

For the simulations in this work, the projection onto the signal space of the virtual channel has been replaced by $\mathbf{H}_{\text {virtual }, i}^{H}$ (a matched filter) resulting in higher signal levels, which improves capacity. Therefore, (8) can be rewritten as

$$
\mathbf{P}_{i}=\tilde{\mathbf{V}}_{\text {null }, i}\left(\mathbf{H}_{i} \tilde{\mathbf{V}}_{\text {null }, i}\right)^{H} .
$$

The Zero-Forcing Tomlinson-Harashima Precoding (ZFTHP) is a nonlinear technique that can be seen as the counterpart of the decision feedback equalizer at the receiver. In a THP system, the already transmitted symbols are fed back in a closed loop and modulo operations, which are denoted by $\mathrm{M}\left({ }^{*}\right)$, are applied at both transmitter and receiver, [10], [11], [12], [13]. The idea behind the THP theory is illustrated in Fig. 2.

The forward and feedback filters are given by

$$
\mathbf{P}=\mathbf{H}^{H} \boldsymbol{\Pi}^{(\mathcal{O}), T} \mathbf{L}^{H,-1} \operatorname{diag}\left(l_{1,1}^{-1}, \ldots, l_{N_{\mathrm{R}}, N_{\mathrm{R}}}^{-1}\right)
$$

and

$$
\mathbf{F}=\mathbf{I}_{B}-\mathbf{L} \operatorname{diag}\left(l_{1,1}^{-1}, \ldots, l_{N_{\mathrm{R}}, N_{\mathrm{R}}}^{-1}\right),
$$

respectively. $\mathbf{L} \in \mathbb{C}^{N_{\mathrm{R}} \times N_{\mathrm{R}}}$ is a lower triangular matrix obtained from the Cholesky decomposition of the channel Gram 

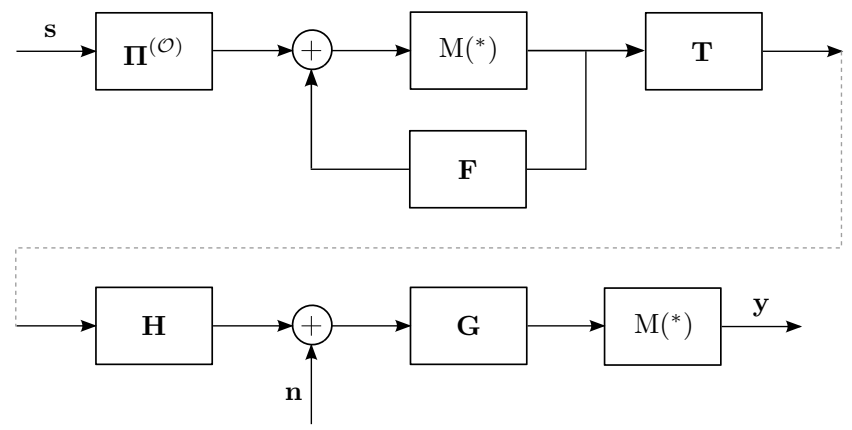

Fig. 2. Block diagram of the THP transmission/reception chain.

matrix $\mathbf{H H}^{H}, l_{i, i}$ is an element of the main diagonal of $\mathbf{L}$, and the receive filter $\mathbf{G}$ is assumed to be a real scalar gain.

The $\Pi^{(\mathcal{O})}$ matrix is responsible for permuting the symbols in such a way that the signal levels at the output of the THP system are maximized. The ordering process considered for the simulations in this work is described in [13], [14].

The QR Decomposition-Based Successive Interference Cancellation (QRSIC) is a nonlinear technique similar to THP in some sense. The basic differences between them is that QRSIC does not perform any ordering and no energy normalization is applied (the term $\operatorname{diag}\left(l_{1,1}^{-1}, \ldots, l_{N_{\mathrm{R}}, N_{\mathrm{R}}}^{-1}\right)$ in (11) forces the energy to be distributed uniformly among all transmit symbols). In addition to that, a QR decomposition is used here rather than a Cholesky one as in THP. This technique is an implementation of the algorithm described in [15]. The forward and feedback filters of the QRSIC algorithm, respectively, are given by

$$
\begin{gathered}
\mathbf{P}=\mathbf{H}^{H} \mathbf{Q}^{H}, \\
\mathbf{F}=\eta\left(\mathbf{I}-\operatorname{diag}\left(r_{1,1}^{-1}, \ldots, r_{N_{\mathrm{R}}, N_{\mathrm{R}}}^{-1}\right) \mathbf{R}\right),
\end{gathered}
$$

where $\mathbf{Q} \in \mathbb{C}^{N_{\mathrm{R}} \times N_{\mathrm{R}}}$ is a unitary matrix and $\mathbf{R} \in \mathbb{C}^{N_{\mathrm{R}} \times N_{\mathrm{R}}}$ is a lower triangular matrix, which are obtained from the $\mathrm{QR}$ decomposition of the channel Gram matrix, $r_{i, i}$ is an element of the main diagonal of $\mathbf{R}$, the receive filter $\mathbf{G}$ is assumed to be a real scalar gain, and $\eta$ is a regularization parameter, which has a role equivalent to that of the $\alpha$ parameter of the linear MMSE algorithm.

\section{Simulations And Results}

Here, a scenario with two different antenna configurations, $(2,2,3,3)$ and $(4,2,3,3)$, is considered. Where the notation $\left(N_{\mathrm{t}}, N_{\mathrm{r}}, N_{\mathrm{b}}, N_{\mathrm{u}}\right)$ indicates a scenario with $N_{\mathrm{b}}$ BSs equipped with $N_{\mathrm{t}}$ transmit antennas each and $N_{\mathrm{u}}$ MSs with $N_{\mathrm{r}}$ receive antennas each. The two receive antennas at each MS considered in the present simulations are assumed to be crosspolarized.

Each of the $N_{\mathrm{b}}$ cooperative BSs is placed in the center of a hexagonal cell with radius of $1000 \mathrm{~m}$. For each channel realization, one user is arbitrarily located in each cell. Rayleigh fading is assumed for small-scale fading. For the large-scale fading, shadow fading is simulated with a variance of $\sigma^{2}=$ $6 \mathrm{~dB}$ and the following path-loss expression is employed:

$$
G_{\mathrm{PL}}=15.3+37.6 \log _{2} d \quad[\mathrm{~dB}],
$$

where $d$ is the distance between BS and MS.

Simulations are performed for a low and a high average SNR (at cell edge), namely $-5 \mathrm{~dB}$ and $20 \mathrm{~dB}$ along with different values for the perturbation variance $\sigma_{e}^{2}$ varying from $40 \mathrm{~dB}$ (negligible perturbation, and thus considered here as the absence of channel estimation error) to $10 \mathrm{~dB}$ (actually this corresponds to an impractical channel error, but is considered so as to visualize the tendency of curves in the graphical results).

The present performance analysis is twofold: an absolute performance evaluation and a sensitivity evaluation. The former is intended to measure the impact of different antenna configurations on the average spectral efficiency per user (in $\mathrm{bps} / \mathrm{Hz}$ ) of each precoder assuming a $20 \mathrm{~dB}$ average SNR at the cell edge in the presence of an unreliable channel estimation, whereas the latter focuses on how much each precoder has its performance diminished as the channel estimation error grows, i.e., how robust they are against fluctuations in channel estimation assuming low and high values for the average SNR at the cell edge $(-5 \mathrm{~dB}$ and $20 \mathrm{~dB}$ respectively).

Figs. 3(a) and 3(b) show the performance of the precoders for two different antenna configurations assuming imperfect channel estimation for different values of perturbation. When comparing both figures, we can see that the linear technique ZF has its performance scaled by $N_{\mathrm{t}}$ for negligible perturbation. The same happens to MMSE, due to the convergence to ZF for high SNR. Although block-diagonalization-based precoding is also a linear processing technique, it does not follow the same behavior as ZF and MMSE concerning the antenna configuration, as it does not decouple the MIMO channels for each BS-MS pair into parallel interference-free subchannels (or near interference-free in the case of MMSE). On the other hand, the BDNSD technique has better performance compared to the other linear techniques. These results also show that this precoder can also outperform nonlinear techniques in the presence of harmful channel perturbation. Another interesting outcome of these results is that, apart from the BDNSD precoder, all the others converge to a common performance for strong channel estimation errors, which implies that under such a harmful perturbation in channel estimation there is no improvement on the increase of antennas in the MIMO structure. For such high error variances, diversity techniques, which do not depend on the quality of the channel estimation, should be preferred.

For a slight-to-moderate error variance it is seen that, apart from the BDNSD algorithm, the ZF-THP is the one that presents the best robustness to channel estimation errors. The advantage of ZF-THP is more visible for the $(2,2,3,3)$ antenna configuration, whereas for the $(4,2,3,3)$ case it becomes less relevant.

Figs. 4(a) and 4(b) show the behavior of each precoder regarding their sensitivity to estimation errors for both low and high SNR values and a $(4,2,3,3)$ antenna configuration. The sensitivity to estimation errors $\Psi\left(\sigma_{\mathrm{e}}^{2}\right)$ is defined as the ratio of the average spectral efficiency per user given a certain error variance $S\left(\sigma_{\mathrm{e}}^{2}\right)$ to the average spectral efficiency per user 


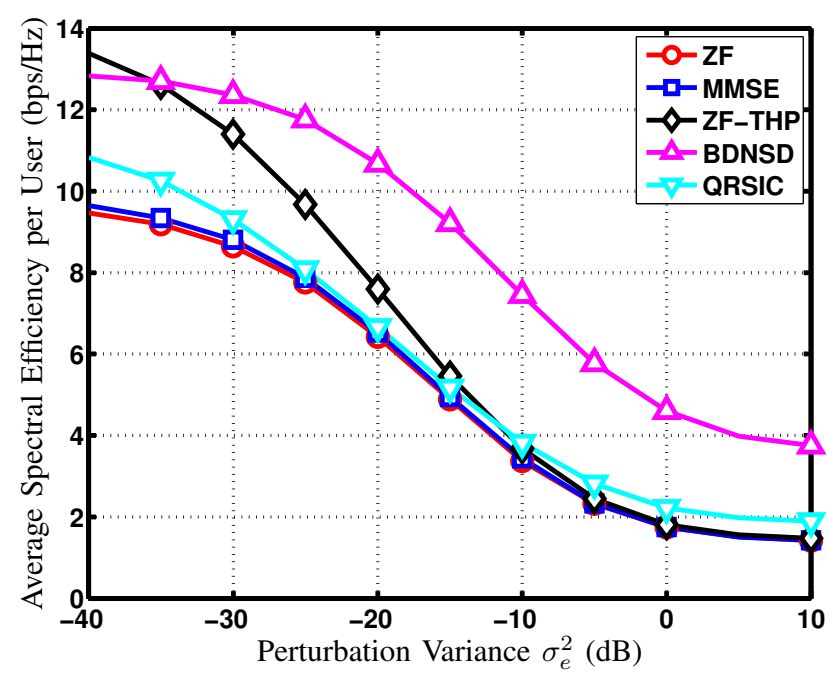

(a) $(2,2,3,3)$ case.

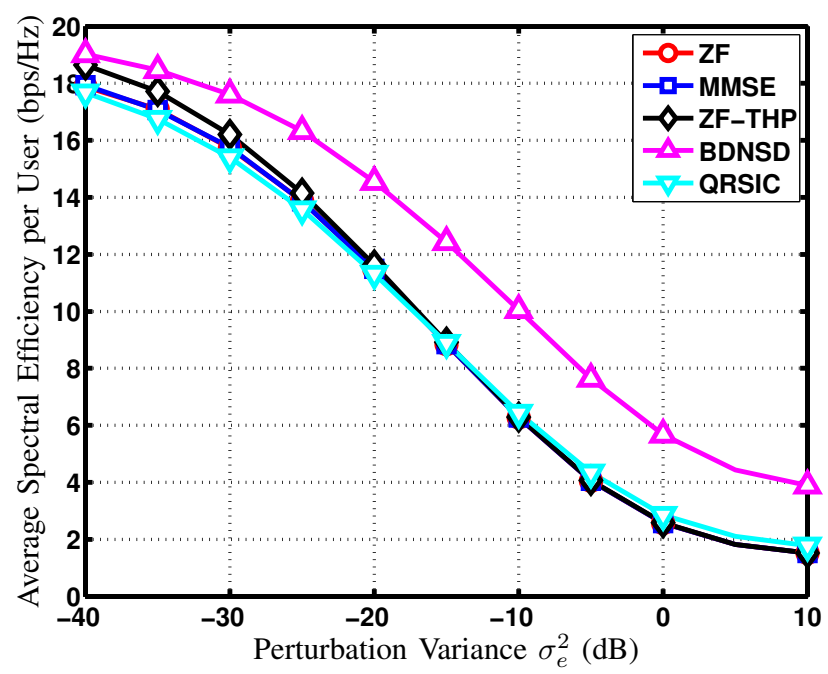

(b) $(4,2,3,3)$ case.

Fig. 3. Performance Curves for a 20dB average SNR at the cell edge and different antenna configurations.

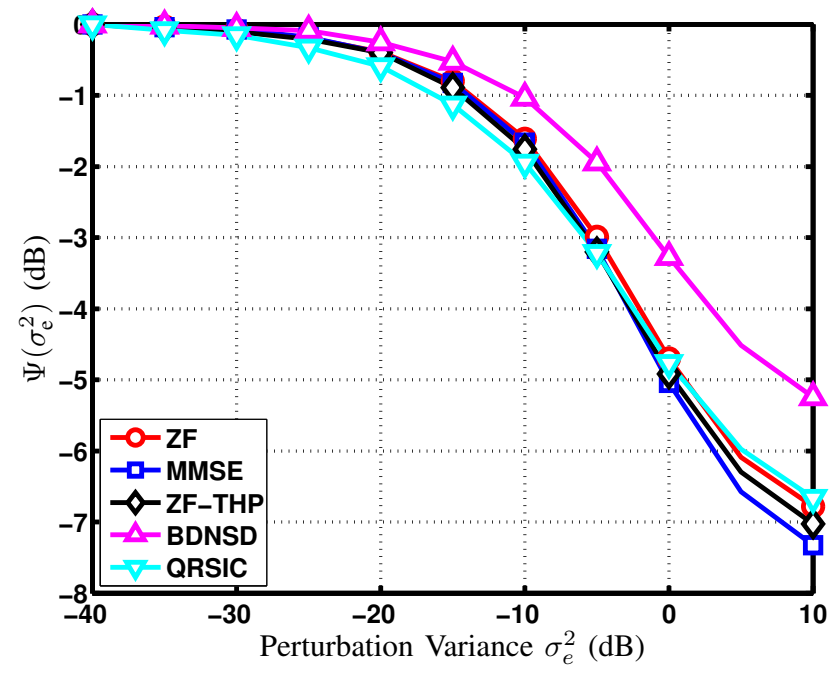

(a) $-5 \mathrm{~dB}$ average $\mathrm{SNR}$ at the cell edge.

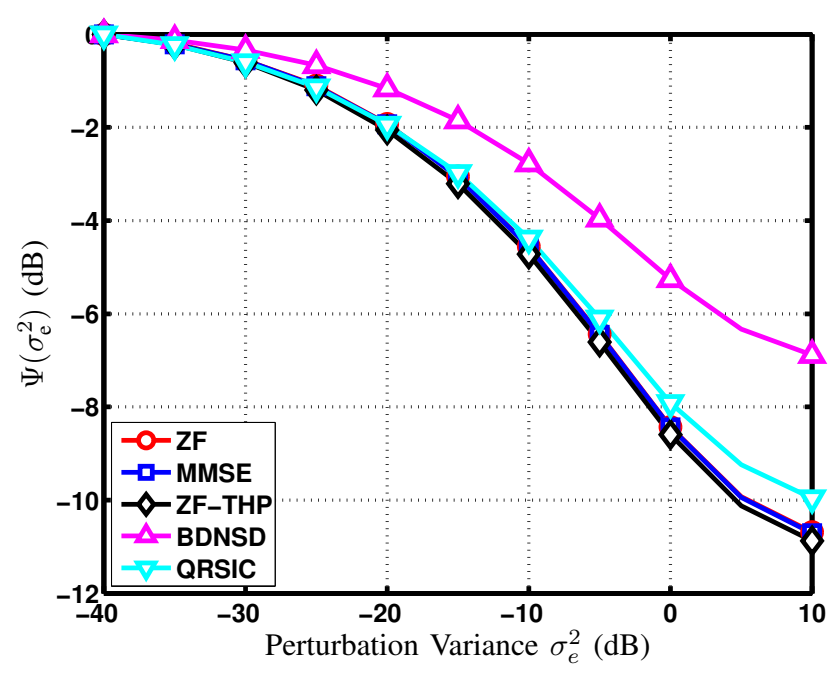

(b) $20 \mathrm{~dB}$ average SNR at the cell edge.

Fig. 4. Sensitivity Analysis for the $(4,2,3,3)$ case and different SNR values at the cell edge.

of the error-free case $S_{0}$, i.e.,

$$
\Psi\left(\sigma_{\mathrm{e}}^{2}\right)=10 \log _{10}\left\{\frac{S\left(\sigma_{\mathrm{e}}^{2}\right)}{S_{0}}\right\} \quad[\mathrm{dB}] .
$$

These results reveal that the BDNSD precoder is the least sensitive in this sense and that all the other precoders seem to behave similarly.

In spite of the apparent advantage of the BDNSD technique over all the other algorithms in terms of error sensitivity, there are some other relevant issues that need to be discussed. Firstly, the BDNSD requires a higher receiver complexity, since the interference among streams of a same user needs to be mitigated at the receiver. Secondly, it has been assumed in the simulations that no estimation errors were made at the receivers. It is therefore expected that, if estimation errors at the receiver are of the same order as those at the transmitter, then the BDNSD algorithm should present a behavior similar to that of the other techniques. For these reasons, the BDNSD algorithm would be more advisable for situations in which the channel estimation at the receivers presents a good reliability.

\section{Conclusions}

Five different precoding techniques have been evaluated in the presence of channel estimation errors in a multicell multiuser MIMO system with the assumption of perfect base station cooperation. It has been shown that the algorithms present different sensitivities to the estimation errors depending on the antenna configuration and on the SNR.

The BDNSD algorithm was shown to present the best robustness to channel estimation errors, but at the cost of increased receiver complexity and assuming perfect channel knowledge at the receivers. If estimation errors are also present at the receivers, its performance is expected to be similar to the other algorithms. It was also seen that, aside from the BDNSD algorithm, the ZF-THP presented the best robustness 
for slight-to-moderate error variances and for the $(2,2,3,3)$ antenna configuration. For strong perturbation variances, with the exception of the BDNSD algorithm, the algorithms converge to the same performance.

\section{ACKNOWLEDGMENTS}

This work was supported by the Research and Development Centre, Ericsson Telecomunicações S.A., Brazil, under EDB/UFC.21 Technical Cooperation Contract. Velser D. B. Correia is scholarship supported by CAPES.

\section{REFERENCES}

[1] G. J. Foschini and M. J. Gans, On limits of wireless communications in a fading environment when using multiple antennas, Wireless Personal Communications, v. 6, n. 3, pp. 331-335, 1998.

[2] A. Goldsmith, S. A. Jafar, N. Jindal, and S. Vishwanath, Capacity Limits on MIMO Channels, IEEE Journal on Selected Areas in Communications, v. 21, n. 5, pp. 684-702, 2003.

[3] D. Chizhik, G. J. Foschini, M. J. Gans, and R. A. Valenzuela, Keyholes, correlations and capacities of multielement transmit and receive antennas, IEEE Transactions on Wireless Communications, v. 1, n. 2, pp. 361-368, 2002.

[4] H. Dai and H. V. Poor, Asymptotic spectral efficiency of multicell MIMO systems with frequency flat fading, IEEE Transactions on Signal Processing, v. 51, n. 11, pp. 2976-2988, 2003.

[5] H. Dai, A. F. Molisch and H. V. Poor, Downlink capacity of interferencelimited MIMO systems with joint detection, IEEE Transactions on Wireless Communications, v. 3, n.2, pp. 442-453, 2004.
[6] H. Zhang and H. Dai, Cochannel Interference Mitigation and Cooperative Processing in Downlink Multicell Multiuser MIMO Networks, EURASIP Journal on Wireless Communications and Networking, v. 2, pp. 222-235, 2004.

[7] G. J. Foschini, K. Karakayali and R. A. Valenzuela, Coordinating multiple antenna cellular networks to achieve enormous spectral efficiency, IEE Proceedings in Communications v. 153, August 2006.

[8] M. Joham and J. A. Nossek, Linear Transmit Processing in MIMO Communications Systems, IEEE Transactions on Signal Processing, v. 53, pp. 2700-2712, August 2005.

[9] Q. H. Spencer and A. L. Swindlehurst and M. Haardt, Zero-forcing methods for downlink spatial multiplexing in multiuser MIMO channels, IEEE Transactions on Signal Processing, v. 52, pp. 461-471, February 2004.

[10] M. Tomlinson, New Automatic Equalizer Employing Modulo Arithmetic, Electronics Letters, v. 7, pp. 138-139, March 1971.

[11] H. Harashima and H. Miyakawa,Matched-Transmission Technique for Channels With Intersymbol Interference, IEEE Transactions on Communications, v. 20, pp. 774-780, August 1972.

[12] R. F. H. Fischer and C. Windpassinger and A. Lampe and J. B. Huber, Space-time transmission using Tomlinson-Harashima precoding, IEEE Transactions on Information Theory, pp. 139-142, January 2002.

[13] M. Joham and W. Utschick, Ordered spatial Tomlinson-Harashima precoding, Chapter 20 of Smart Antennas - State of the Art, EURASIP Book Series on Signal Processing and Communications, v. 3, Hindawi Publishing Corporation, 2005.

[14] M. Joham and D. A. Schmidt and H. Brunner and W. Utschick, A symbol-wise order optimization for successive precoding, in Proc. ITG/IEEE Workshop on Smart Antennas (WSA), February 2007.

[15] C. B. Peel, Studies in Multiple-antenna wireless communications, $\mathrm{PhD}$ thesis, Brigham Young University, April 2004. 